\title{
FLOW RATE INSENSITIVE THERMAL CONDUCTIVITY DETECTOR
}

\author{
B.C. Kaanta ${ }^{l}, H$. Chen ${ }^{2}$, and X. Zhang ${ }^{l *}$ \\ ${ }^{1}$ Boston University, Boston, MA, USA \\ ${ }^{2}$ Schlumberger Doll Research, Cambridge, MA, USA
}

\begin{abstract}
We present a novel thermal conductivity detector design in which output is minimally affected by changing flow rates in a microchannel. In gas chromatography systems, pressure fluctuations can result in false peaks and an unstable baseline, reducing the systems limit of detection. Furthermore, ramping the temperature of a separation column, a method used in many chemical separations, changes the viscosity and therefore the flow rate of the carrier gas, resulting in an unstable baseline. Therefore, the development of a flow rate invariant detector is extremely important in reducing the complexity and increasing the portability of gas chromatography systems.
\end{abstract}

\section{INTRODUCTION}

\section{$\mu$ Gas Chromatography}

Rapid detection of gas phase chemicals is critical in a number of wide-ranging applications, such as environmental monitoring, industrial process control, public safety and point of care health services. The miniaturization of gas chromatography (GC) devices has greatly increased the portability and the number of applications for this powerful analytical tool. However, further advancements can be made to increase the simplicity and portability of these systems.

Gas chromatography systems make it possible to identify and quantify compounds of interest by using a column to separate individual components in space and time. The sample is transported along the length of the separation column in a carrier gas, commonly helium. Interaction between the sample and a stationary phase in the column physically delays the elution of the components to different degrees based on their chemical properties. Temperature ramping is often used to increase the speed of elution of heaver chemical components [1,2]. These separated gas components are subsequently passed through a detector and quantified.

Traditionally, carrier gas flow perturbations in a thermal conductivity detector's (TCD) signal has been accounted for by using a second TCD as a reference cell. However, this can double the consumable requirements (such as carrier gas) and the number of fluidic connections, as well as introducing numerous additional potential sources of error. Ideally, a TCD should not be affected by changing flow velocity.

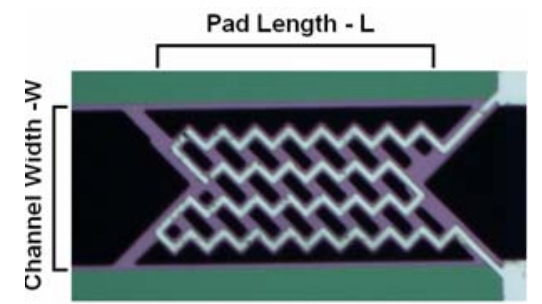

Figure 1: Single element $\mu T C D$. Power is applied to device and only the average temperature over the whole length of the pad can be detected.

\section{$\mu$ Thermal Conductivity Detector Models}

As the gas sample passes over a $\mu \mathrm{TCD}$, power dissipation in the filament causes joule heating, increasing the electrical resistance of the filament. Filament resistance is directly related the detector temperature. Heat is carried away from a suspended heater element in 4 ways; conduction along the supports, conduction through the gas, mass transport by moving gas, and radiation $[2,3]$.

A TCD signal comes from the magnitude of the conductive heat flux between the heated detector element and the channel walls, via the gas surrounding the element. The three other heat loss pathways are not affected by the makeup of the sample gas.

To date, all $\mu$ TCDs have been composed of a single resistive heating element.(Figure 1) When a single element is exposed to a moving gas stream, the leading edge cools down, changing the detector output. However, the thermodynamic models of planar TCDs hypothesize that if an element is held at a constant temperature, the central region will be flow invariant $[5,6]$. The technical challenge has been to design a TCD which can maintain a fixed temperature along the entire structure.

\section{MODELING AND SIMULATION}

To achieve a flow rate independent detector, our new device is composed of several electrically and mechanically independent active regions, each maintained at a constant temperature (Figure 2). This represents a significant enhancement on our previously reported high sensitivity thermal conductivity detectors [7].

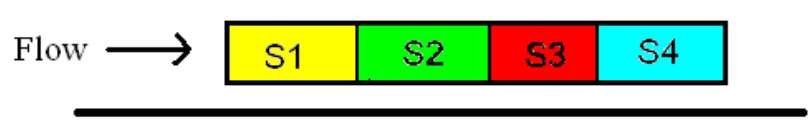

Figure 2: TCD with multiple independent active regions S1-S4. $S 1$ preheats the flow. S2-4 is in a fully developed thermal region and responds only to the changing thermal conductivity of the gas surrounding it.

The central region of the flat filament is flow independent because it is past the thermal entry region of the pad and the thermal gradient is fully developed.(Figure 3) Once the isothermal lines are parallel to the hot filament, the heat is transported through the surrounding gas as if it were stationary, effectively decoupling mass transport from the heat flux equation. The upstream heating element is used to generate this stable thermal gradient for the following detector elements.

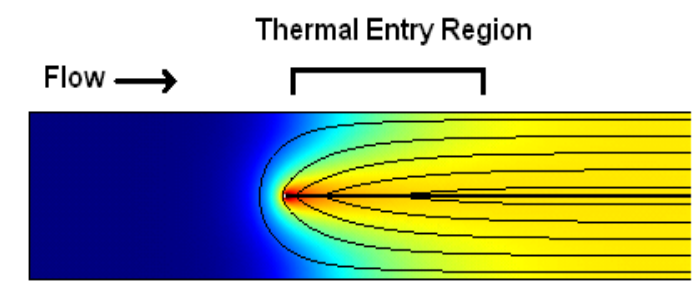

Figure 3: Simulation of heat flux showing temperature isotherm development after the flow contacts the heater pad. 'Thermal Entry Region' is where the isotherms are not parallel to flow and convection cannot be discounted as a heat flow pathway.

Work has been done in modeling the thermal performance of a $\mu$ TCD. Finite element modeling tests confirm some of the 
theoretical work of Huang \& Bau [5], and Chen \& Wu [6], which concluded that heat loss from forced convection, is proportional to thermal entry length. However, their calculations were based on the assumption that the heater pad completely isothermal, which is invalid for most situations, especially for physical single element TCDs.

Modeling and finite element simulations have been performed to calculate the effects of changing flow rate on a series of closely spaced constant temperature $\mu$ TCDs as opposed to a single constant temperature element. Figure 4 shows the results from a model of four $500 \mu \mathrm{m}$ heater pads with $20 \mu \mathrm{m}$ spacing between pads placed in a $100 \mu \mathrm{m}$ tall channel. The model was created using a symmetry plain though the center of the heater pads. As the flow rate increases, more and more power is required to maintain TCD1 at a fixed temperature. As the flow is increased from $0.5 \mathrm{~m} / \mathrm{s}$ to $3 \mathrm{~m} / \mathrm{s}$, all of these gas velocities are in the laminar flow regime. However, TCD2 and TCD3 are almost completely isolated from the effects of the changing flow rate up to $2 \mathrm{~m} / \mathrm{s}$. At $3 \mathrm{~m} / \mathrm{s}, \mathrm{TCD} 2$ is affected as the thermal boundary layer is no longer completely generated by the end of TCD1. TCD4 experiences some tailing effects at lower flow rates, as it is required to heat the area downstream of the structure.

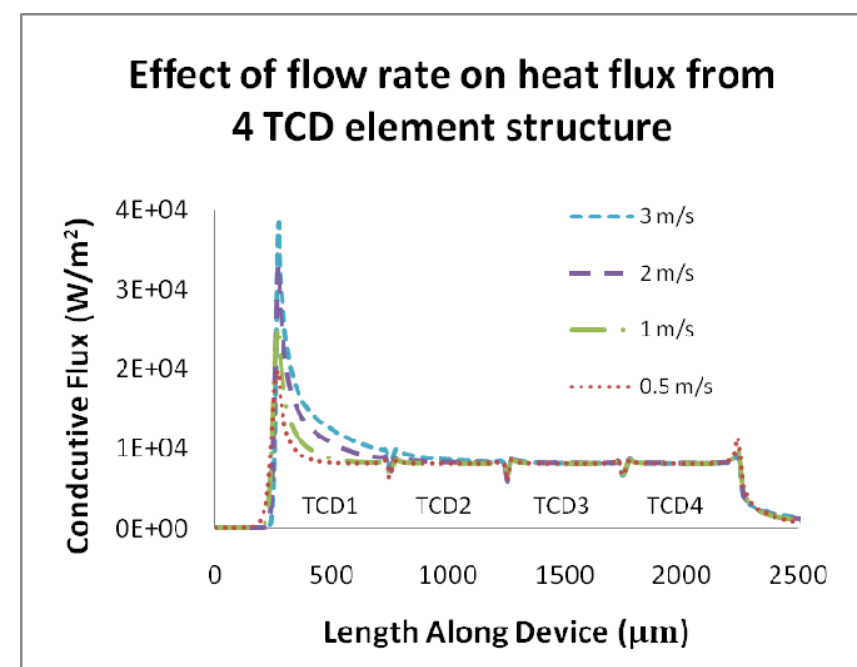

Figure 4: FEM model of heat flux from TCDs in a micro channel at 4 different flow rates, flow moving from left to right. All TCDs are set to an equal constant temperature. TCD1 is much more affected by changing flow rate then other elements.

\section{FABRICATION}

The presented multiple element $\mu \mathrm{TCD}$ was fabricated by depositing nickel over a chromium adhesion layer. This metal layer was supported on a $0.5 \mu \mathrm{m}$ silicon nitride film $(\mathrm{SiN})$. Before the metal layer was deposited, the SiN layer was pre-etched $150 \mathrm{~nm}$ in the pattern of the mask for the metal layer. The metal was then deposited, filling these pre-etched grooves (Figure 5a). This process creates a flush surface, which is critical for bonding a sealing cover over the flow channel. The nickel layer connects contact pads with wide traces to $5 \mu \mathrm{m}$-wide resistive heaters.

a

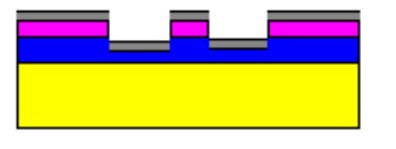

b
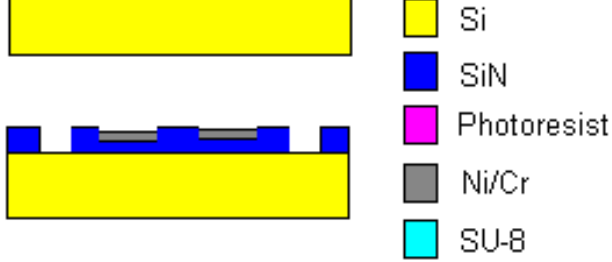

C

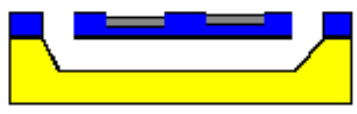

d

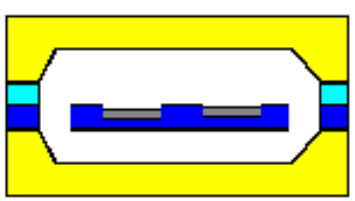

Figure 5: Device fabrication sequence for suspended $\mu T C D$. a) Pre-Etch of SiN and lift-off of metal layer. b) Etch of SiN film to define heater pad and channels. c) Release of heater pad with $\mathrm{KOH}$ etch. d) Device is sealed with silicon covers using a polymer intermediate layer.

After lift-off, the flow channels and support pad forming the resistive heating element were defined by patterning and etching through the SiN layer (Figure $5 \mathrm{~b}$ ). The structure was released using an anisotropic potassium hydroxide $(\mathrm{KOH})$ etch. The channel was sealed by bonding a cover, which also had a channel etched using the same $\mathrm{KOH}$ process. A SU-8 intermediate layer was used bond the two chips together. Alignment and bonding between the device and cover chip was performed using an FC150 Flip Chip Bonder. Two versions of the multiple element $\mu \mathrm{TCD}$ were fabricated, with pad length as the only variable parameter. Completed device before bonding with 4 electrically independent TCD's is shown in figure 6.

After the channels were sealed, fused silica capillary tubing was inserted into the channels and sealed in place using an epoxy. Leak testing was performed by pressurizing the device and placing it in a water bath to check for bubbles.

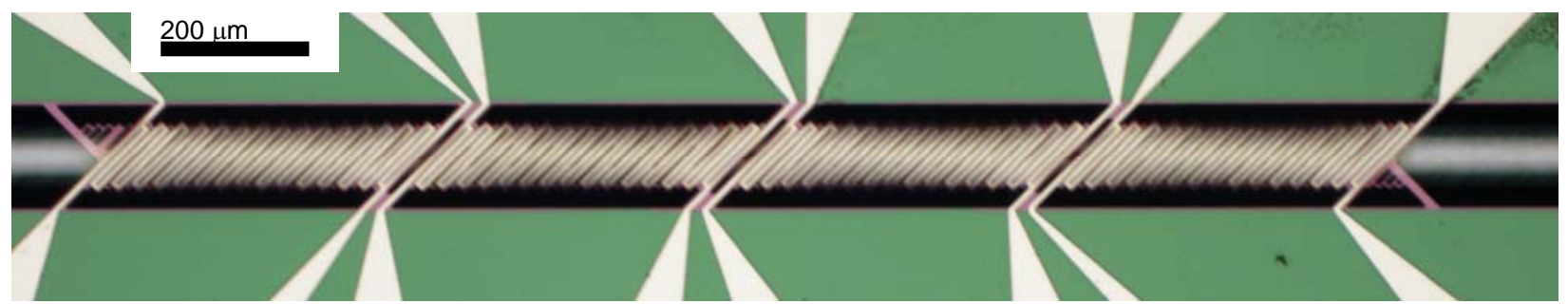

Figure 6: The optical image of 4 electrically independent high sensitivity thermal conductivity detectors .The power input and temperature of each detector can be controlled and monitored independently. 


\section{TESTING SETUP \& CALIBRATION}

After fabrication, each 4 element device was tested for continuity and thermally calibrated by placing it in a temperaturecontrolled oven and measuring the resistance of each element as the oven temperature was increased. This thermal calibration was used to calculate the resistance the $\mu$ TCDs must be set to for any given operating temperature.

Each of the four sections used a constant temperature control circuit to maintain its $\mu \mathrm{TCD}$ element at a set resistance (Figure 7). The resistance (and therefore the temperature) of each independent section was maintained by a current source with a feedback loop. This circuit used a reference resistance and adjusted the current by resistive heating until the resistance of the $\mu \mathrm{TCD}$ matched the reference resistance. The analog output of each control circuit was digitized using a National Instruments analog to digital converter and recorded on a computer.

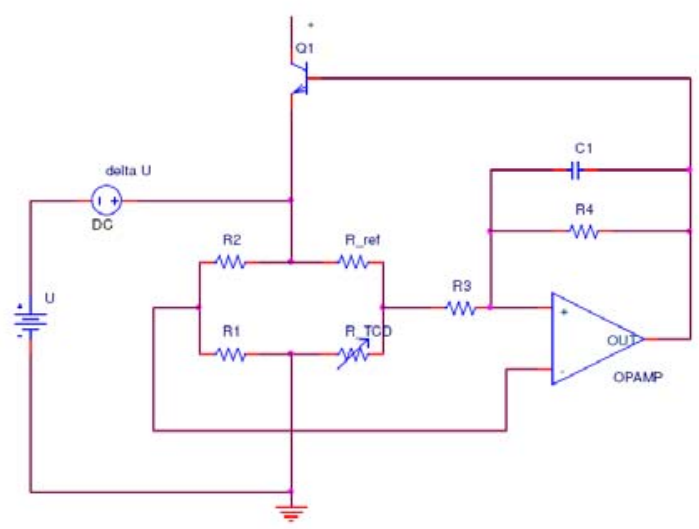

Figure 7: Simple constant temperature feedback circuit. By adjusting $R \_$ref, each TCD can be set at an operating temperature. Top of the Wheatstone bridge, delta $U$, is the output signal from the detector.

Flow rate testing was performed using the pressure controller from an Agilent Technologies 7890A Gas Chromatography System. This pressure controller made it possible to ramp the inlet pressure between 1 and 90psi at a 60psi per minute. Original trials used a 5 meter capillary column between the pressure controller and the $\mu$ TCDs. However, this setup failed to achieve a sufficiently high flow rate to ever effect the second or third TCD in the flow path. The column was removed, drastically decreasing the fluidic restriction and increasing the flow rates through the detector. The flow rates achieved in this setup are more than 10 times greater than those used in normal GC systems.

\section{TESTING AND RESULTS}

\section{Flow Rate Independence}

Two different 4 element $\mu$ TCDs were tested. The difference between the devices was the length of the $\mu \mathrm{TCD}$ elements in the channel, one contained four $\sim 500 \mu \mathrm{m} \mu \mathrm{TCDs}$ and the other contained four $\sim 1000 \mu \mathrm{m} \mu \mathrm{TCDs}$ in series. The testing was performed in a temperature-controlled convection oven maintained at $30^{\circ} \mathrm{C}$. The thermal calibration data was used to select the set point resistance so that the temperature of all four $\mu$ TCDs would be $60^{\circ} \mathrm{C}$ which was $30^{\circ} \mathrm{C}$ over the ambient environment.

Both devices showed a significant reduction in the response of the downstream $\mu$ TCDs to changing flow rates (Table 1). Furthermore, the device with the shorter $\mu \mathrm{TCD}$ pads showed a larger effect and earlier breakdown of flow insensitivity for the trailing pads. This fit with the modeled expectations. Both devices matched our modeling and showed that the third TCD was least effected by changing flow rates. This is because the last TCD in the flow path is expected to experience tailing effects.

Table 1: Maximum response of $\mu T C D$ sections as a percentage of the change in the leading TCD section (TCD1) at 90psi. TCD 1 is the leading upstream section follow in order by TCD2, TCD3, and TCD4.

\begin{tabular}{|l|c|c|c|}
\hline Device & TCD2 & TCD3 & TCD4 \\
\hline $500 \mu \mathrm{m}$ TCDs & $14.61 \%$ & $3.22 \%$ & $4.25 \%$ \\
\hline $1000 \mu \mathrm{m}$ TCDs & $5.75 \%$ & $0.27 \%$ & $1.40 \%$ \\
\hline
\end{tabular}

Figures 8 and 9 show the TCD response plotted against the system head pressure. The downstream elements in the device with the shorter $\mu$ TCDs began have a significant response at around 50psi.

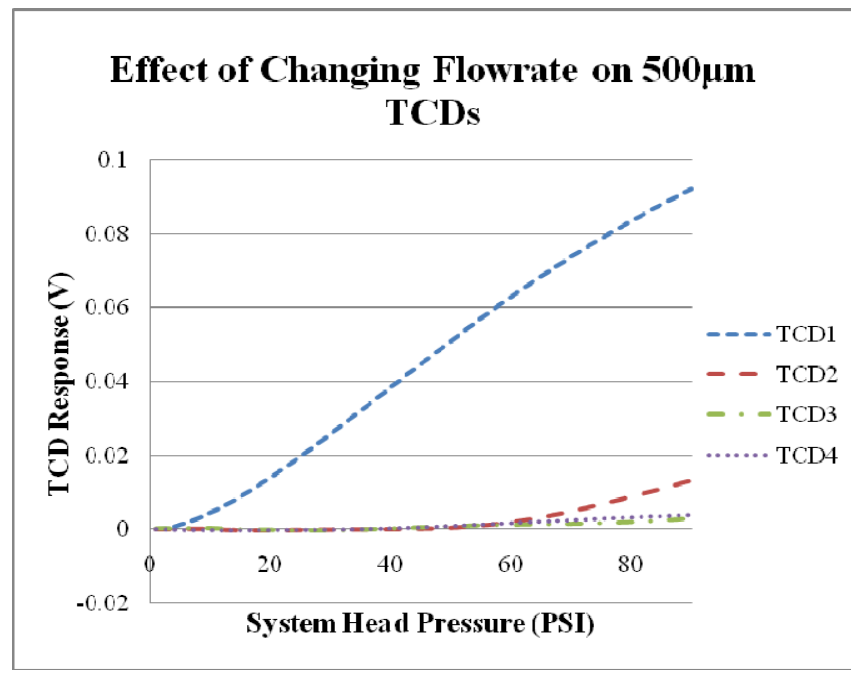

Figure 8: Effect of ramped input pressure on the four $500 \mu \mathrm{m}$ elements. TCD1 is clearly the most affected with TCD2 beginning to be affected at $50 \mathrm{psi}$.

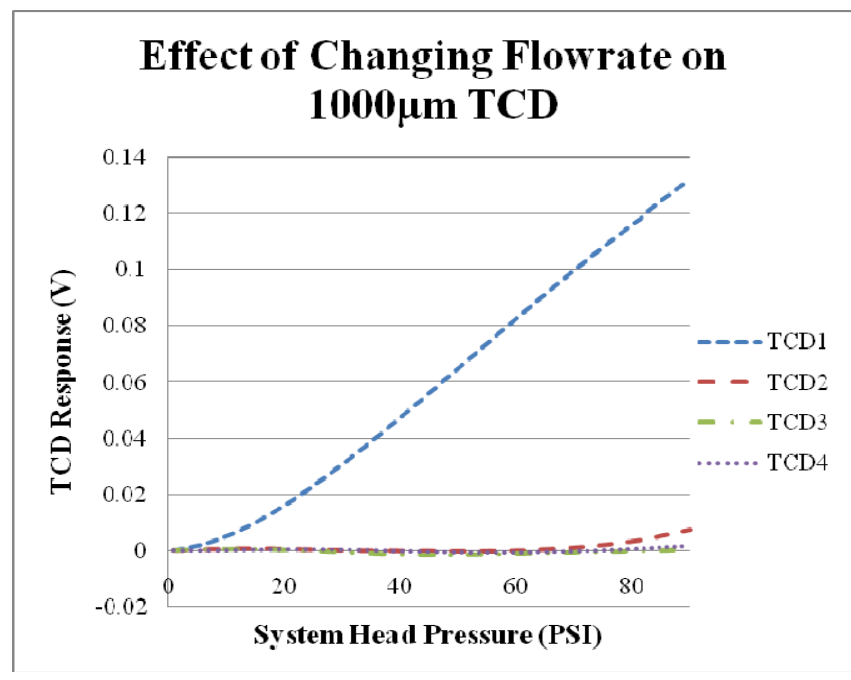

Figure 9: Effect of ramped input pressure on the four $1000 \mu \mathrm{m}$ elements. TCD1 is clearly the most affected with TCD2 beginning to be affected at around 70psi. 
In the device with the longer $\mu \mathrm{TCD}$ elements, power dissipation from the second $\mu \mathrm{TCD}$ only began to increase with a head pressure of $70 \mathrm{psi}$.

These tests show that the thermal entry region causes flow rate variation in the output of the detector. Longer preheating regions result in a broader range of flow independence for the detector.

In both devices, the $\mu$ TCDs $2-4$ had a small negative response (required less power to maintain the set point temperature). This is most likely because of errors in the set point resistance of the individual $\mu \mathrm{TCD}$ elements. All TCDs were suppose to be set to $60^{\circ} \mathrm{C}$, however, if an upstream TCD was set hotter than $60^{\circ} \mathrm{C}$, then the isotherms would be distorted and as the flow rate increases the power required for the following TCD to maintain its set temperature will decrease. Work is being continued to improve the calibration of the $\mu$ TCDs and the control and stability of the set point to try and minimize these problems.

\section{TCD Interdependence}

To show that the close spacing of the $\mu$ TCD elements was key to the flow rate insensitivity, a test was performed only powering the first and third $\mu$ TCDs. In this test, the two active TCDs where separated by $\sim 500 \mu \mathrm{m}$. The two devices showed nearly identical responses up to $20 \mathrm{psi}$ head pressure. This implies that no heat from the first element was reaching the second element $500 \mu \mathrm{m}$ downstream. After 20psi, the third $\mu \mathrm{TCD}$ element was affected by the first $\mu \mathrm{TCD}$; however, at the maximum tested system head pressure, the third $\mu \mathrm{TCD}$ only required $55 \%$ of the power used by the first $\mu \mathrm{TCD}$ to maintain its set point temperature (Figure 10).

The first element has the same response as with all four $\mu$ TCDs powered which shows there is little or no thermal crosstalk up stream in the carrier gas flow.

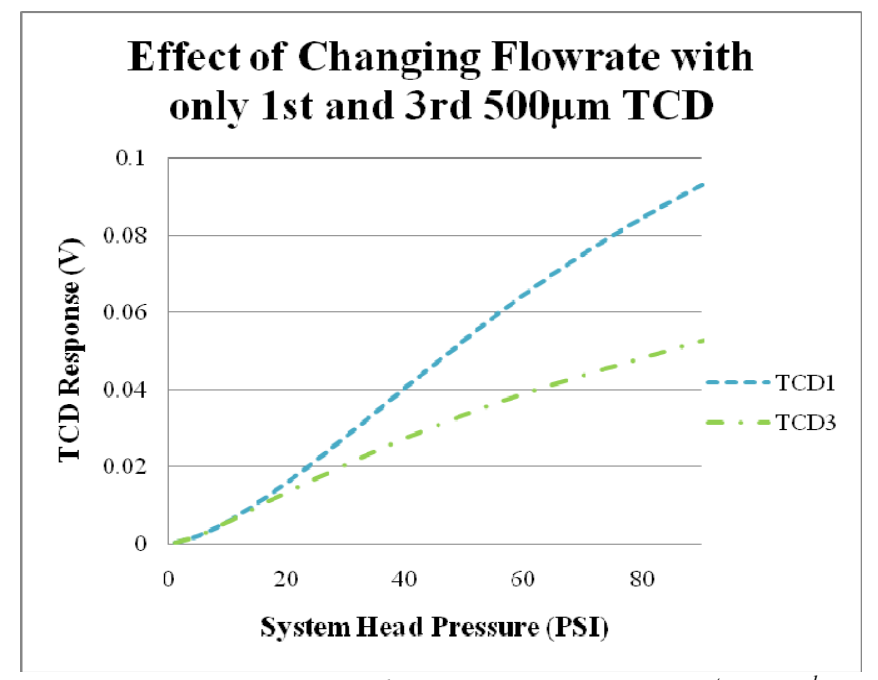

Figure 10: Effect of ramped input pressure on the $1^{\text {st }}$ and $3^{\text {rd }}$ $500 \mu \mathrm{m}$ elements in device. TCD1 and TCD3 have similar responses up to 20psi. After 20psi heat from TCD1 affect TCD3.

\section{CONCLUSION}

The design, fabrication and testing of several $\mu$ TCDs with a high degree of independence to carrier gas flow have been shown. The heat flux in the system has been modeled and tested experimentally to increase understanding of microchannel thermodynamics and improving the device performance. Initial testing has shown no noticeable loss of sensitivity in the detection of analytes for these multiple element $\mu$ TCDs as compared to a traditional single element $\mu$ TCD. Combining this detector with a micro-fabricated separation column and injector would create a complete $\mu \mathrm{GC}$ system with a simpler design, fewer interconnections and lower gas consumption requirements.

\section{ACKNOWLEDGEMENTS}

We would like to acknowledge National Science Foundation (ECCS 0901702), Schlumberger-Doll Research and the Boston University Photonics Center for their support of this research.

\section{REFERENCES}

[1] M. Agah et al., "High-Performance Temperature-Programmed Microfabricated Gas Chromatography Columns," J. Microelectromech. Syst. Vol. 14, pp. 1039-1050, 2005

[2] J. Potkay, et al., "A Low-Power Pressure- and TemperatureProgrammable Micro Gas Chromatography Column," J. Microelectromech. Syst. Vol. 16, pp. 1071-1079, 2007

[3] Y.E. Wu, K. Chen, C.W. Chen, K.H. Hsu, "Fabrication and Characterization of Thermal Conductivity Detectors (TCDs) of Different Flow Channel and Heater Designs", Sensors and Actuators A, 100, 37, (2002).

[4] Chen, K., and Wu, Y. E., "Thermal Analysis and Simulation of the Microchannel Flow in Miniature Thermal Conductivity Detectors," Sensors and Actuators A, vol. 79, pp. 211-218, 2000.

[5] Y. Huang, H. H. Bau, "The Effects of Forced Convection on the Power Dissipation of Constant-Temperature Thermal Conductivity Sensors," Transactions of the ASME, Vol. 119, 1997.

[6] Y. M. Kim, W. S Kim, W. G. Chun, "A Numerical Study of the Thermal Entrance Effect in Miniature Thermal Conductivity Detectors", Heat Transfer Engineering, 26, 65 (2005).

[7] B.C. Kaanta et al, "High sensitivity micro-thermal conductivity detector for gas chromatography," Proc. IEEE MEMS 2009, (2009), pp. 264.

\section{CONTACT}

*X. Zhang, tel: +1-617-358-2702; xinz@bu.edu 